\title{
THE ENERGY OPERATOR FOR A MODEL WITH A MULTIPARAMETRIC INFINITE STATISTICS
}

\author{
Stjepan Meljanac ${ }^{1}$, Ante Perica ${ }^{1}$ \\ and Dragutin Svrtan ${ }^{2}$ \\ ${ }^{1}$ Rudjer Bošković Institute - Bijenička c. 54, 10000 Zagreb, Croatia \\ 2 Dept. of Math., Univ. of Zagreb, Bijenička c. 30, 10000 Zagreb, Croatia
}


Abstract. In this paper we consider energy operator (a free Hamiltonian), in the second-quantized approach, for the multiparameter quon algebras: $a_{i} a_{j}^{\dagger}-q_{i j} a_{j}^{\dagger} a_{i}=$ $\delta_{i j}, i, j \in I$ with $\left(q_{i j}\right)_{i, j \in I}$ any hermitian matrix of deformation parameters. We obtain an elegant formula for normally ordered (sometimes called Wick-ordered) series expansions of number operators (which determine a free Hamiltonian). As a main result (see Theorem 1) we prove that the number operators are given, with respect to a basis formed by "generalized Lie elements", by certain normally ordered quadratic expressions with coefficients given precisely by the entries of the inverses of Gram matrices of multiparticle weight spaces. (This settles a conjecture of two of the authors (S.M and A.P), stated in [8]). These Gram matrices are hermitian generalizations of the Varchenko's matrices, associated to a quantum (symmetric) bilinear form of diagonal arrangements of hyperplanes (see [12]). The solution of the inversion problem of such matrices in [9] (Theorem 2.2.17), leads to an effective formula for the number operators studied in this paper.

The one parameter case, in the monomial basis, was studied by Zagier [15], Stanciu [11] and Møller [6].

PACS numbers: 03.65.-w, 05.30.-d, 02.20.Uw

Key words: Infinite statistics, multiparameter deformations 
1. Introduction. One-parameter quonic intermediate statistics [2][3][4], which interpolate between Bose-Einstein and Fermi-Dirac statistics, are examples of infinite statistics in which any representation of the symmetric group can occur. These models offer a possibility of a small violation of the Pauli exclusion principle, at least in nonrelativistic theory [3][5]. In a seminal paper [15], Zagier made an explicit computation of the Gram determinants of multiparticle weight spaces of the Fock representation (which for $q \in\langle-1,1\rangle$ proves a Hilbert space realizability of "q-mutator relations " $\left.a_{i} a_{j}^{\dagger}-q a_{j}^{\dagger} a_{i}=\delta_{i j}, i, j \in I\right)$ and begun a study of particle number operators. A slight variation of the Zagier's conjecture [15] on the form of a normally ordered series expansion of the number operators in a monomial basis is proved subsequently by Stanciu in [11]. Generally, physical observables in the second-quantized approach are represented in terms of creation and annihilation operators in the normally ordered form (see Møller [6]). Meljanac and Perica started (in [7], [8]) with an idea to extend the above results to the multi-parameter case: $a_{i} a_{j}^{\dagger}-q_{i j} a_{j}^{\dagger} a_{i}=\delta_{i j}, i, j \in I$, where each commutation relation has its own deformation parameter $q_{i j}$ (a complex number) satisfying $q_{j i}=\left(q_{i j}\right)^{*}$ (where ${ }^{*}$ denotes complex conjugation).

Subsequently, in [9] (see also [10]) two types of results are proved:

Ad.1. In case of distinct quantum numbers the multi-parameter Gram determinants (Theorem 1.9.2) are computed by extending Zagier's method, which in turn gives also a hermitian analogue of the Varchenko's determinant of the (symmetric) quantum bilinear form of diagonal arrangements of hyperplanes. From this explicit computation a Hilbert space realizability follows in case when all $\left|q_{i j}\right|<1$ (cf. other 
methods presented in [16] and [17]).

Ad.2. Explicit formulas (Theorem 2.2.17) are obtained for the inverse of the Gram matrices of arbitrary multiparticle weight spaces, by following ideas of Božejko and Speicher (given in [16]). In particular, a counterexample (when $n=8$ ) to a conjecture of Zagier (also stated in [15]), for the form of the inverse in the one-parameter case, is found. In [9] an appropriate extension of Zagier's conjecture for the form of the inverse of multi-parameter Gram matrices is also formulated and proved.

In this paper we study number operators (and hence energy operator) in the spirit of the second-quantized approach. The approach is basically algebraic, i.e. independent of any particular representation (see Greenberg [3], Møller [6], Stanciu [11], Meljanac and Perica [8]).

The main result of this paper is the Theorem 1, in which we show that the coefficients of the normally ordered series expansion of particle number operators in the Fock representation, in terms of a basis of "generalized Lie elements", are given precisely by certain inverse matrix entries of the Gram matrices on the multiparticle weight spaces. This confirms a conjecture of Meljanac and Perica in [8]. Thus, in conjunction with the results of [9], one obtains explicit expression for the number operators in multiparametric quon algebras.

\section{Multi-parameter quon algebras and Gram matrices.}

Let $\mathbf{q}=\left\{q_{i j}: i, j \in I,\left(q_{i j}\right)^{*}=q_{j i}\right\}$ be a hermitian family of complex numbers (parameters), where $I$ is a finite (or infinite) set of indices. Recall that (cf. [9]) by a multiparameter quon algebra $\mathcal{A}=\mathcal{A}^{(\mathbf{q})}$ we shall mean an associative (complex) algebra generated by $\left\{a_{i}, a_{i}^{\dagger}, i \in I\right\}$ subject to the following $q_{i j^{-}}$canonical commutation 
relations:

$$
a_{i} a_{j}^{\dagger}=q_{i j} a_{j}^{\dagger} a_{i}+\delta_{i j}, \quad \forall i, j \in I
$$

The algebra $\mathcal{A}$ has a cannonical anti-involution ${ }^{\dagger}: \mathcal{A} \rightarrow \mathcal{A}$ (which exchanges $a_{i}$ with $a_{i}^{\dagger}$, reverses products and on the coefficients acts by complex conjugation.)

Recall that a Fock representation of $\mathcal{A}$ is given by a family of linear operators $a_{i}: \mathcal{H} \rightarrow \mathcal{H}$ on a complex Hilbert space $\mathcal{H}, i \in I$, satisfying the following canonical commutation(or " $q_{i j}$-mutator") relations:

$$
\begin{gathered}
a_{i} a_{j}^{\dagger}-q_{i j} a_{j}^{\dagger} a_{i}=\delta_{i j}, i, j \in I \\
a_{i}|0\rangle=0, i \in I
\end{gathered}
$$

where $a_{i}^{\dagger}$ denotes the adjoint of $a_{i}$, and $|0\rangle$ denotes a distinguished ("vacuum") vector in $\mathcal{H}$.

Any total order on the indexing set $I$ induces a total order on the set $I^{*}$ of all sequences (=words) $\mathbf{i}=i_{1} \ldots i_{n}$ over $I$. Then we can consider the Gram matrix

$$
A=(\langle\mathbf{i} \mid \mathbf{j}\rangle)
$$

of all $n$-particle states $|\mathbf{i}\rangle:=a_{i_{1}}^{\dagger} a_{i_{2}}^{\dagger} \cdots a_{i_{n}}^{\dagger}|0\rangle\left(i_{j} \in I, n \geq 0\right)$. Its entries $\langle\mathbf{i} \mid \mathbf{j}\rangle$ are the "expectation values" (i.e. overlaps of $n$-particle states in the second quantized Fock description)

$$
\left\langle 0\left|a_{i_{n}} \cdots a_{i_{1}} a_{j_{1}}^{\dagger} \cdots a_{j_{m}}^{\dagger}\right| 0\right\rangle
$$

These entries vanish, unless (i) $n=m$ and (ii) $i_{1} \cdots i_{n}$ and $j_{1} \cdots j_{m}$ are permutations of the same weakly increasing sequences $\nu=k_{1} \ldots k_{n}, k_{1} \leq \cdots \leq k_{n},\left(k_{j} \in I\right)$, which we shall call weights. Thus the matrix $A$ is block diagonal (cf. [9,Proposition 1.6.1]):

$$
A=\oplus_{n \geq 0} \oplus_{k_{1} \leq \cdots \leq k_{n}} A^{k_{1} \ldots k_{n}}
$$


with blocks $A^{\nu}=A^{k_{1} \ldots k_{n}}$ indexed by weights. The size of $A^{\nu}$ is equal to the number of permutations (or rearrangements) of the multiset $\left\{k_{1} \leq \cdots \leq k_{n}\right\}$.

For $\nu=k_{1}<k_{2}<\cdots<k_{n}$ ( a generic weight ), $A^{\nu}$ is a matrix of order $n$ ! with rows/columns labelled by rearrangements (of $\nu$ ) $\mathbf{i}=i_{1} \ldots i_{n}=k_{\pi(1)} \ldots k_{\pi(n)}=: \nu . \pi$, ( $\pi \in S_{n}=$ the $n$-th symmetric group) or simply by permutations $\pi \in S_{n}$. The entry of $A^{\nu}$ in the row $\mathbf{i}=\nu . \pi$ and column $\mathbf{j}=\nu . \sigma$ is then given explicitly by the following formula:

$$
A_{\mathbf{i}, \mathbf{j}}^{\nu}=A^{\nu}(\pi, \sigma)=\prod_{(r, s) \in I\left(\sigma^{-1} \pi\right)} q_{k_{\pi(r)} k_{\pi(s)}}
$$

where, for $\pi \in S_{n}, I(\pi)$ denotes the set of inversions of $\pi: I(\pi)=\{(r, s): 1 \leq r<$ $s \leq n, \pi(r)>\pi(s)\}$. Thus, we can view $A^{\nu}$ as a linear operator on the group algebra $\mathbf{C}\left[S_{n}\right]=\left\{\sum_{\pi \in S_{n}} c_{\pi} \pi: c_{\pi} \in \mathbf{C}, \pi \in S_{n}\right\}$

For general weights $\tilde{\nu}=\left(\tilde{k}_{1}=\cdots=\tilde{k}_{n_{1}}<\tilde{k}_{n_{1}+1}=\cdots=\tilde{k}_{n_{1}+n_{2}}<\cdots<\right.$ $\left.\tilde{k}_{n_{1}+\cdots n_{p-1}+1}=\cdots=\tilde{k}_{n}\right), n_{1}+n_{2}+\cdots+n_{p}=n$, the matrix $A^{\tilde{\nu}}$ has order equal to $n ! / n_{1} ! \cdots n_{p}$ ! and its rows/columns are labelled by rearrangements $\mathbf{i}=i_{1} \ldots i_{n}=\tilde{\nu} . \tilde{\pi}$, $\tilde{\pi} \in H_{\tilde{\nu}} \backslash S_{n}$, where $H_{\tilde{\nu}}=\operatorname{Stab}_{\tilde{\nu}}=\left\{\sigma \in S_{n} \mid \tilde{\nu} . \sigma=\tilde{\nu}\right\}$ is the (stabilizer) subgroup fixing $\tilde{\nu}$. The $(\mathbf{i}, \mathbf{j})$-th entry of $A^{\tilde{\nu}}, \mathbf{i}=\tilde{\nu} . \tilde{\pi}, \mathbf{j}=\tilde{\nu} . \tilde{\sigma}, \tilde{\pi}=H \pi, \tilde{\sigma}=H \sigma$, where $\pi, \sigma$ are unique coset representatives (of minimal length) of $\tilde{\pi}, \tilde{\sigma}$, is given by

$$
A_{\mathbf{i}, \mathbf{j}}^{\tilde{\nu}}=A^{\tilde{\nu}}(\tilde{\pi}, \tilde{\sigma})=\sum_{\tau \in \tilde{\sigma}^{-1} \tilde{\pi}=\sigma^{-1} H \pi} \prod_{(r, s) \in I(\tau)} q_{i_{r} i_{s}}=\sum_{\tau \in \sigma^{-1} H \pi} \prod_{(r, s) \in I(\tau)} q_{k_{\pi(r)} k_{\pi(s)}}
$$

(Note that $(\tilde{5})$ generalizes $(5)$, because $\operatorname{Stab}_{\nu}=H_{\nu}=\{1\}$, if $\nu$ is generic.) In $\left[9\right.$,Subsection 1.7] it is shown that the operator $A^{\tilde{\nu}}$ can be obtained from $A^{\nu}(\nu=$ $k_{1}<\ldots<k_{n}$ ) by a reduction procedure in two steps: first by identifying indices $k_{1} \mapsto$ $\tilde{k_{1}}, \ldots, k_{n} \mapsto \tilde{k_{n}}$ and then restricting this specialized operator $\left.A^{\nu}\right|_{\nu \mapsto \tilde{\nu}}$ to the invariant 
subspace (in $\mathbf{C}\left[S_{n}\right]$ ) spanned by $H_{\tilde{\nu}}$ - invariant vectors $\bar{\sigma}=\sum_{h \in H_{\tilde{\nu}}} h \sigma \in \mathbf{C}\left[S_{n}\right]$. In fact $(\tilde{5})$ can be rewritten as

$$
A^{\tilde{\nu}}(\tilde{\pi}, \tilde{\sigma})=\left.\sum_{h \in H_{\tilde{\nu}}} A^{\nu}(\pi, h \sigma)\right|_{\nu \mapsto \tilde{\nu}}
$$

As a consequence we obtain : if $\left.A^{\nu}\right|_{\nu \mapsto \tilde{\nu}}$ is invertible, then the matrix $A^{\tilde{\nu}}$ is invertible too, and a relation analogous to (6) holds for the inverses. In particular, $\operatorname{det} A^{\tilde{\nu}}$ $\left.\operatorname{divides} \operatorname{det} A^{\nu}\right|_{\nu \mapsto \tilde{\nu}}$. This shows that in order to study some properties (e.g. invertibility or positive definiteness) it suffices to consider the generic case (when all the indices $k_{i}$ are distinct).

Now we list some properties of the matrices $A^{\nu}, \nu=k_{1}<k_{2} \cdots<k_{n}$ :

$$
\begin{gathered}
(a) \quad A^{\nu}(\pi, \pi)=1 \\
\text { (b) } A^{\nu}(\sigma, \pi)=A^{\nu}(\pi, \sigma)^{*} \text { (hermiticity) }
\end{gathered}
$$

(c) Let $w_{n}=n \ldots 21$ be the longest permutation in $S_{n}$. Then

$$
A^{\nu}\left(\pi w_{n}, \sigma w_{n}\right)=A^{\nu}(\sigma, \pi)=A^{\nu}(\pi, \sigma)^{*}
$$

The property c) can be rewritten in the matrix form as follows :

$$
W A^{\nu} W=\left(A^{\nu}\right)^{T}, \quad W^{2}=1
$$

where

$$
W(\pi, \sigma)=\left\{\begin{array}{l}
1, \text { if } \pi w_{n}=\sigma \\
0, \text { otherwise }
\end{array}\right.
$$

It is important to note that the Fock space, in our case, is positive definite iff the Gram matrix $A$ is positive definite. Recall that a sufficient condition for the positivity of norm squared of all vectors is (cf [9, Theorem 1.9.4])

$$
\left|q_{i j}\right|<1, \quad \forall i, j \in I
$$


In particular, the condition (12) implies that the $n$-particle states $|\mathbf{i}\rangle=a_{i_{1}}^{\dagger} \cdots a_{i_{n}}^{\dagger}|0\rangle$ $\left(i_{j} \in I, n \geq 0\right)$ are linearly independent.

Examples: For the generic weights $\nu=1,12,123$ the Gram matrices are as follows:

$$
A^{1}=(1) ; \quad A^{12}=\left(\begin{array}{cc}
1 & q_{12} \\
q_{21} & 1
\end{array}\right)
$$

$A^{123}=$\begin{tabular}{|c|c|c|c|c|c|c|}
\hline$\pi \backslash \sigma$ & 123 & 132 & 312 & 321 & 231 & 213 \\
\hline 123 & 1 & $q_{23}$ & $q_{13} q_{23}$ & $q_{12} q_{13} q_{23}$ & $q_{12} q_{13}$ & $q_{12}$ \\
\hline 132 & $q_{32}$ & 1 & $q_{13}$ & $q_{12} q_{13}$ & $q_{12} q_{13} q_{32}$ & $q_{12} q_{32}$ \\
\hline 312 & $q_{31} q_{32}$ & $q_{31}$ & 1 & $q_{12}$ & $q_{12} q_{32}$ & $q_{12} q_{31} q_{32}$ \\
\hline 321 & $q_{21} q_{31} q_{32}$ & $q_{21} q_{31}$ & $q_{21}$ & 1 & $q_{32}$ & $q_{31} q_{32}$ \\
\hline 231 & $q_{21} q_{31}$ & $q_{21} q_{31} q_{23}$ & $q_{21} q_{23}$ & $q_{23}$ & 1 & $q_{31}$ \\
\hline 213 & $q_{21}$ & $q_{21} q_{23}$ & $q_{21} q_{13} q_{23}$ & $q_{13} q_{23}$ & $q_{13}$ & 1 \\
\hline
\end{tabular}

(here we use the Johnson-Trotter ordering of permutations:123,132,312,321,231,213).

For the non-generic: $\tilde{\nu}=11,113$, the Gram matrices are:

$$
A^{11}=\left(1+q_{11}\right) ; \quad A^{113}=\begin{array}{|c|c|c|c|}
\hline \pi \backslash \sigma & 113 & 131 & 311 \\
\hline 113 & 1+q_{11} & q_{13}+q_{11} q_{13} & q_{13}^{2}+q_{11} q_{13}^{2} \\
\hline 131 & q_{31}+q_{11} q_{31} & 1+q_{11} q_{13} q_{31} & q_{13}+q_{11} q_{13} \\
\hline 311 & q_{31}^{2}+q_{11} q_{31}^{2} & q_{31}+q_{11} q_{31} & 1+q_{11} \\
\hline
\end{array}
$$

The inverses of the Gram matrices in the generic case above, are given by:

$$
\left[A^{12}\right]^{-1}=\frac{1}{\Delta^{12}}\left(\begin{array}{cc}
1 & -q_{12} \\
-q_{21} & 1
\end{array}\right)=\frac{1}{\Delta^{12}}\left(\begin{array}{cc}
1 & q_{12} \\
q_{21} & 1
\end{array}\right) *\left(\begin{array}{cc}
1 & -1 \\
-1 & 1
\end{array}\right)
$$

where $\Delta^{12}:=1-q_{12} q_{21}=1-\left|q_{12}\right|^{2}$, and

$$
\left[A^{123}\right]^{-1}=\frac{1}{\Delta^{123}} A^{123} * M^{123}
$$

Here $\Delta^{123}:=\left(1-\left|q_{12}\right|^{2}\right)\left(1-\left|q_{13}\right|^{2}\right)\left(1-\left|q_{23}\right|^{2}\right)\left(1-\left|q_{12}\right|^{2}\left|q_{13}\right|^{2}\left|q_{23}\right|^{2}\right), *$ denotes the Schur product of matrices $\left(a_{i j}\right) *\left(b_{i j}\right):=\left(a_{i j} b_{i j}\right)$ and $M^{123}$ stands for the following 
matrix:

\begin{tabular}{|c|c|c|c|c|c|c|}
\hline$\pi^{\backslash \sigma}$ & 123 & 132 & 312 & 321 & 231 & 213 \\
\hline 123 & $(1-a c)(1-b)$ & $(b-1)(1-c)$ & $c(b-1)(1-a)$ & $(1-a c)(1-b)$ & $a(b-1)(1-c)$ & $(b-1)(1-a)$ \\
\hline 132 & $(c-1)(1-b)$ & $(1-a b)(1-c)$ & $(c-1)(1-a)$ & $a(c-1)(1-b)$ & $(1-a b)(1-c)$ & $b(c-1)(1-a)$ \\
\hline 312 & $(a-1)(1-b)$ & $(a-1)(1-c)$ & $(1-b c)(1-a)$ & $(a-1)(1-b)$ & $b(a-1)(1-c)$ & $(1-b c)(1-a)$ \\
\hline 321 & $(1-a c)(1-b)$ & $a(b-1)(1-c)$ & $(b-1)(1-a)$ & $(1-a c)(1-b)$ & $(b-1)(1-c)$ & $c(b-1)(1-a)$ \\
\hline 231 & $a(c-1)(1-b)$ & $(1-a b)(1-c)$ & $b(c-1)(1-a)$ & $(c-1)(1-b)$ & $(1-a b)(1-c)$ & $(c-1)(1-a)$ \\
\hline 213 & $(a-1)(1-b)$ & $b(a-1)(1-c)$ & $(1-b c)(1-a)$ & $c(a-1)(1-b)$ & $(a-1)(1-c)$ & $(1-b c)(1-a)$ \\
\hline
\end{tabular}

(with $a:=\left|q_{23}\right|^{2}, b:=\left|q_{13}\right|^{2}, c:=\left|q_{12}\right|^{2}$ ).

The inverse in the non-generic case $\nu=113$ is given by

$$
\left[A^{113}\right]^{-1}=\frac{1}{\Delta^{113}}\left(\begin{array}{ccc}
1 & -\left(1+q_{11}\right) q_{13} & q_{11} q_{13}^{2} \\
-q_{31}\left(1+q_{11}\right) & \left(1+q_{11}\right)\left(1+q_{13} q_{31}\right) & -\left(1+q_{11}\right) q_{13} \\
q_{31}^{2} q_{11} & -q_{31}\left(1+q_{11}\right) & 1
\end{array}\right)
$$

where $\Delta^{113}=\left(1+q_{11}\right)\left(1-q_{13} q_{31}\right)\left(1-q_{11} q_{13} q_{31}\right)=\left(1+q_{11}\right)\left(1-\left|q_{13}\right|^{2}\right)\left(1-q_{11}\left|q_{13}\right|^{2}\right)$.

\section{Series expansions of number operators.}

First we recall that the $k$-th particle number operator $N_{k}(k \in I$ ) (in the Fock representation satisfying the positivity condition (12)) is a diagonal operator which counts the number of appearances of the creation operator $a_{k}^{\dagger}$ in any multi-particle state $|\mathbf{i}\rangle$. These operators satisfy the following implicit conditions (equations):

$$
\begin{gathered}
{\left[N_{k}, a_{l}\right]=-a_{k} \delta_{k l}, \quad \forall k, l \in I} \\
N_{k}|0\rangle=0, \quad \forall k \in I
\end{gathered}
$$

Note that for any fixed $k \in I$, if we assume (12), the equations (13) have unique solution for $N_{k}$. The number operators play an important role in constructing the free Hamiltonian (= the energy operator) of the free system (for which the energy is additive, cf. Greenberg [3]) of generalized quon particles in the nonrelativistic limit:

$$
H=\sum_{k \in I} E_{k} N_{k}
$$


More generally, our primary goal here is to express $N_{k}$ in terms of quon algebra generators as an normally ordered infinite series involving certain iterated deformed commutators of the creation and annihilation operators.

It is already indicated in [8] that the formal expansion of the number operator $N_{k}$ in terms of normally ordered products is necessarily of the following form which preserves each $n$-particle subspace (it easily follows from (3)):

$$
N_{k}=\sum_{\mathbf{i} \in I^{+}, i_{1}=k} X_{\mathbf{i}}^{\dagger} Y_{\mathbf{i}}
$$

where $I^{+}$denotes the set of all nonempty words (or sequences) $\mathbf{i}=i_{1} \ldots i_{n}, n \geq 1$ over the set $I$ as an alphabet, and the sum is over those words which begin with letter $k$. Here, if the indices $i_{1}, \ldots, i_{n}$ are distinct, we require that $X_{\mathbf{i}}$ and $Y_{\mathbf{i}}$ are both multihomogeneous of the same multidegree, i.e. they are expressible as a linear combination of all rearrangements $a_{\mathbf{j}}=a_{\mathbf{i}} \cdot \pi:=a_{\mathbf{i} . \pi}\left(=a_{i_{\pi(1)}} a_{i_{\pi(2)}} \cdots a_{i_{\pi(n)}}\right)$ of the "monomial" $a_{\mathbf{i}}=a_{i_{1}} a_{i_{2}} \cdots a_{i_{n}}$, in the following form:

$$
\begin{aligned}
& X_{\mathbf{i}}=\sum_{\pi \in S_{n}} a_{\mathbf{i} . \pi} x_{\mathbf{i} . \pi, \mathbf{i}} \\
& Y_{\mathbf{i}}=\sum_{\pi \in S_{n}} a_{\mathbf{i} . \pi} y_{\mathbf{i} \cdot \pi, \mathbf{i}}
\end{aligned}
$$

where $x_{\mathbf{i} . \pi, \mathbf{i}}$ and $y_{\mathbf{i} . \pi, \mathbf{i}}$ are, yet unknown, coefficients (depending on $q_{i j}$ 's) with the following normalization convention $y_{\mathbf{i}, \mathbf{i}}=1$. For general i' $\mathrm{s}$, we require that the summations in (16) and (17) should be replaced by summations over the left cosets $H \backslash S_{n}$, where $H=\mathrm{Stab}_{\mathbf{i}}$ is the stabilizer subgroup of $S_{n}$ fixing $\mathbf{i}$, with coefficients 
$\tilde{x}_{\mathbf{i} . \tilde{\pi}, \mathbf{i}}, \tilde{y}_{\mathbf{i} . \tilde{\pi}, \mathbf{i}}, \tilde{\pi} \in H \backslash S_{n}$ equal to the following orbit sums:

$$
\begin{aligned}
\tilde{x}_{\mathbf{i} . \tilde{\pi}, \mathbf{i}} & =\sum_{h \in H} x_{\mathbf{i} . h \pi, \mathbf{i}} \\
\tilde{y}_{\mathbf{i} . \tilde{\pi}, \mathbf{i}} & =\sum_{h \in H} y_{\mathbf{i} . h \pi, \mathbf{i}}
\end{aligned}
$$

Now we start finding the solution of the system (13), in the form (15), as follows: We first use the fact that under the condition (12), the set of all monomials $a_{i_{n}}^{\dagger} \cdots a_{i_{1}}^{\dagger} a_{j_{1}} \cdots a_{j_{m}},\left(i_{k}, j_{l} \in I\right)$ is linearly independent.Then, we plug the right hand side of (15) into the system(13). By resolving it successively in degree one, then in degree two, etc., we obtain the following (noncommutative) recursions for $Y_{\mathbf{i}}$ 's:

RECURSIONS FOR Y's:

$$
Y_{i_{1} i_{2} \cdots i_{n}}=Y_{i_{1} \cdots i_{n-1}} a_{i_{n}}-q_{i_{n} i_{1}} q_{i_{n} i_{2}} \cdots q_{i_{n} i_{n-1}} a_{i_{n}} Y_{i_{1} \cdots i_{n-1}}
$$

and similarly, a system of "twisted" partial differential equations for $X_{\mathbf{i}}$ 's:

EQUATIONS FOR $X$ 's:

$$
{ }_{l} \partial\left(X_{i_{1} \cdots i_{n}}\right)^{\dagger}=\left(X_{i_{1} \cdots i_{n-1}}\right)^{\dagger} \delta_{l i_{n}} \quad\left(l \in\left\{i_{1}, \ldots, i_{n}\right\}\right)
$$

where ${ }_{l} \partial$ denotes the left twisted derivative:

$$
{ }_{l} \partial\left(a_{j_{1}}^{\dagger} \cdots a_{j_{n}}^{\dagger}\right)=\sum_{\left(p: j_{p}=l\right)} q_{l j_{1}} \cdots q_{l j_{p-1}} a_{j_{1}}^{\dagger} \cdots \widehat{a_{j_{p}}^{\dagger}} \cdots a_{j_{n}}^{\dagger}
$$

( ${ }^{\wedge}$ denotes the omission of the corresponding creation operator).

PROPOSITION 1. The Y-components (17) of the solution (15) of eq.(13) are given by the following iterated $\mathbf{q}$-commutator ("generalized Lie elements") formula:

$$
\begin{gathered}
Y_{i_{1}}=a_{i_{1}} \\
Y_{i_{1} i_{2} \ldots i_{n}}=\left[\cdots\left[\left[a_{i_{1}}, a_{i_{2}}\right]_{q_{i_{2} i_{1}}}, a_{i_{3}}\right]_{q_{i_{3} i_{1}} q_{i_{3} i_{2}}}, \ldots, a_{i_{n}}\right]_{q_{i_{n} i_{1}} q_{i_{n} i_{2}} \cdots q_{i_{n} i_{n-1}}}
\end{gathered}
$$

where $[x, y]_{q}=x y-q y x$ denotes the $q$-commutator of $x$ and $y .\left(F o r N_{k}\right.$ we need to set $\left.i_{1}=k\right)$. 
Proof: By iterating (19).

In order to express the formula (22)(and some others later) in the operator form we shall now introduce a twisted group algebra of the permutation group.

\section{A twisted group algebra action.}

Let us consider

(1)A right action of the symmetric group $S_{n}$, by permuting factors of any degree $\mathrm{n}$ monomial in the annihilation operators:

$$
a_{\mathbf{i}} \cdot \pi=\left(a_{i_{1}} a_{i_{2}} \cdots a_{i_{n}}\right) \cdot \pi:=a_{i_{\pi(1)}} a_{i_{\pi(2)}} \cdots a_{i_{\pi(n)}}
$$

(2)A "diagonal" action of the formal power series ring $K_{n}=\mathbf{C}\left[\left[Q_{k, l}, 1 \leq k, l \leq n\right]\right]$ (where $Q_{k, l}$ are commuting indeterminates) defined by:

$$
a_{\mathbf{i}} \cdot Q_{k, l}\left(=\left(a_{i_{1}} a_{i_{2}} \cdots a_{i_{n}}\right) \cdot Q_{k, l}\right):=q_{i_{k} i_{l}} a_{i_{1}} a_{i_{2}} \cdots a_{i_{n}}
$$

(here $q_{i j}$ 's are complex numbers from the canonical commutation relations (1)!). These two actions give rise to an action of a twisted group algebra:

$$
\mathcal{K}_{n}=K_{n}{ }^{\sim}\left[S_{n}\right]
$$

of $S_{n}$ (with coefficients in $K_{n}$ ). The multiplication in the algebra $\mathcal{K}_{n}$ is defined by imposing the following comutation relations ("an action of $S_{n}$ on the coefficient ring $\left.K_{n} "\right)$

$$
\pi Q_{k, l}=Q_{\pi(k) \pi(l)} \pi
$$

It is clear that, by specializing $Q_{k, l}=q(1 \leq k, l \leq n)$, the twisted group algebra $K_{n}{ }^{\sim}\left[S_{n}\right]$ is mapped onto the ordinary group algebra $\mathbf{C}[[q]]\left[S_{n}\right]$ in which, according 
to Zagier [15], live certain important elements : $\alpha_{n}, \beta_{n}, \gamma_{n}, \delta_{n}$ satisfying

$$
\alpha_{n}=\alpha_{n-1} \beta_{n}, \beta_{n}=\delta_{n} \gamma_{n}^{-1}\left(\Rightarrow \alpha_{n}=\beta_{2} \cdots \beta_{n}=\delta_{2} \gamma_{2}^{-1} \delta_{3} \gamma_{3}^{-1} \cdots \gamma_{n-1}^{-1} \delta_{n} \gamma_{n}^{-1}\right)
$$

(Note that our notation for $\delta_{n}$ is shifted by 1 compared with [15], which seems to be more natural!)

These elements, via the regular representation $R_{n}$, were crucial in Zagier's computation of the determinant and the inverse of the one-parameter matrices $A_{n}=A_{n}(q)=$ $R_{n}\left(\alpha_{n}\right)$. We shall now define a "lifting" to $K_{n}^{\sim}\left[S_{n}\right]$ of the Zagier's elements by first defining, for each permutation $\pi \in S_{n}$, an element $\tilde{\pi} \in K_{n}{ }^{\sim}\left[S_{n}\right],\left(\pi \in S_{n}\right)$, which encodes all inversions of $\pi$ :

$$
\tilde{\pi}:=Q_{\pi} \pi, \quad \text { where } \quad Q_{\pi}:=\prod_{1 \leq k<l \leq n, \pi(k)>\pi(l)} Q_{\pi(k), \pi(l)}
$$

with the multiplication rule

$$
\tilde{\sigma} \tilde{\pi}=\left(\prod_{(a, b) \in I(\sigma) \cap I\left(\pi^{-1}\right)} Q_{\sigma(a), \sigma(b)} Q_{\sigma(b), \sigma(a)}\right) \widetilde{\sigma \pi} .
$$

(Observe that $\tilde{\pi}$ generalizes $q^{i(\pi)} \pi, i(\pi):=$ the number of inversions of $\pi$ ).

Then we define a "lifting" of all Zagier's elements by the following formulas:

$$
\begin{aligned}
& \tilde{\alpha}_{n}:=\sum_{\pi \in S_{n}} \tilde{\pi} \\
& \tilde{\beta}_{n}:=\sum_{k=1}^{n} \tilde{t}_{k, n} \\
& \tilde{\gamma}_{n}:=\left(1-\tilde{t}_{1, n}\right)\left(1-\tilde{t}_{2, n}\right) \cdots\left(1-\tilde{t}_{n-1, n}\right) \\
& \tilde{\delta}_{n}:=\left(1-\tilde{t}_{n-1} \tilde{t}_{1, n}\right)\left(1-\tilde{t}_{n-1} \tilde{t}_{2, n}\right) \cdots\left(1-\tilde{t}_{n-1} \tilde{t}_{n-1, n}\right)
\end{aligned}
$$

Similarly we define

$$
\tilde{\alpha}_{n_{1}, n_{2}, \ldots, n_{k}}:=\sum_{\pi \in S_{n_{1}} \times S_{n_{2}} \times \cdots \times S_{n_{k}}} \tilde{\pi}
$$


(Here $t_{k, l}$ denotes the cycle $\left(\begin{array}{cccc}k & k+1 & \cdots & l \\ l & k & \cdots & l-1\end{array}\right) \in S_{n}$ and $t_{k}:=t_{k, k+1}$.) It is easy to check that the following relations, analogous to (27), hold true:

$$
\tilde{\alpha}_{n}=\tilde{\alpha}_{n-1} \tilde{\beta}_{n}, \tilde{\beta}_{n}=\tilde{\delta}_{n} \tilde{\gamma}_{n}^{-1}\left(\Rightarrow \tilde{\alpha}_{n}=\tilde{\beta}_{2} \cdots \tilde{\beta}_{n}=\tilde{\delta}_{2} \tilde{\gamma}_{2}^{-1} \tilde{\delta}_{3} \tilde{\gamma}_{3}^{-1} \cdots \tilde{\gamma}_{n-1}^{-1} \tilde{\delta}_{n} \tilde{\gamma}_{n}^{-1}\right)
$$

Important note. Now we can realize all Gram matrices $A^{\nu}$ from (4) as the matrices of the right multiplication by the lifted Zagier element $\tilde{\alpha}_{n}$ on the space monomials $a_{\mathbf{i}}$ of weight $\nu$. This explains why we needed to introduce a twisted group algebra in the multiparameter case.

In what follows, we shall also need the following notations:

$$
\begin{aligned}
Q_{\{\pi\}}: & =\prod_{1 \leq k<l \leq n, \pi(k)>\pi(l)} Q_{\pi(k), \pi(l)} Q_{\pi(l), \pi(k)} \quad\left(\text { for any } \pi \in S_{n}\right), \\
Q_{T}: & =\prod_{k \neq l \in T} Q_{k, l}(\text { for any set } T \subseteq\{1,2, \ldots, n\})
\end{aligned}
$$

together with the following Lemma which we shall use in the proof of the main result:

LEMMA 1. We have the following identity in $\mathcal{K}_{n}$ :

$$
\tilde{\alpha}_{n-1,1}\left(1-\tilde{t}_{n-1} \tilde{t}_{1, n}\right)=\xi_{n} \tilde{\alpha}_{1, n-2,1}
$$

where $\xi_{n}:=\sum_{k=1}^{n-1}\left(1-Q_{\{k, k+1\}} \cdots Q_{\{k, n\}}\right) \tilde{t}_{1, k}$.

(Recall from (29a) that $\tilde{\alpha}_{n-1,1}=\sum_{\pi \in S_{n-1} \times S_{1}} \tilde{\pi}, \tilde{\alpha}_{1, n-2,1}=\sum_{\pi \in S_{1} \times S_{n-2} \times S_{1}} \tilde{\pi}$.)

Proof: By definition $\tilde{\alpha}_{n-1,1}=\sum_{\pi \in S_{n-1} \times S_{1}} \tilde{\pi}$. By using a factorization $\pi=t_{1, k} \sigma$, where $\pi(1)=k, \sigma \in S_{1} \times S_{n-2} \times S_{1}$, we get $\tilde{\alpha}_{n-1,1}=\left(\sum_{k=1}^{n-1} \tilde{t}_{1, k}\right) \tilde{\alpha}_{1, n-2,1}$ (here we 
used that $\tilde{\pi}=\tilde{t}_{1, k} \tilde{\sigma}$, c.f.(28)). Similarly,

$$
\begin{aligned}
\tilde{\alpha}_{n-1,1} \tilde{t}_{n-1} \tilde{t}_{1, n} & =\sum_{\pi \in S_{n-1} \times S_{1}} \tilde{\pi} \tilde{t}_{n-1} \tilde{t}_{1, n}=\sum_{\pi \in S_{n-1} \times S_{1}} \tilde{\pi} Q_{\{n-1, n\}} \tilde{t}_{1, n-1} \quad(b y(28)) \\
& =\sum_{\pi \in S_{n-1} \times S_{1}} Q_{\{\pi(n-1), \pi(n)\}} \tilde{\pi} \tilde{t}_{1, n-1} \\
& =\sum_{\pi \in S_{n-1} \times S_{1}} Q_{\{\pi(n-1), n\}} Q_{\left\{t_{\pi(n-1), n-1}^{-1}\right\}} \tilde{\pi} t_{1, n-1} \quad(\text { by }(28) \operatorname{and}(34)) \\
& =\sum_{\sigma \in S_{1} \times S_{n-2} \times S_{1}} Q_{\left\{t_{\pi(n-1), n}^{-1}\right\}} \tilde{t}_{1, \pi(n-1)} \tilde{\sigma}\left[t_{1, \pi(n-1)} \sigma=\pi t_{1, n-1}\right] \\
& =\left(\sum_{k=1}^{n-1} Q_{\left\{t_{k, n}^{-1},\right.} \tilde{t}_{1, k}\right) \tilde{\alpha}_{1, n-2,1} .
\end{aligned}
$$

By subtracting the last two formulas, the Lemma follows.

Now we state the formula (22) in the operator form:

COROLLARY 1. We have

i) $Y_{i_{1} \cdots i_{n}}=\left(a_{i_{1}} a_{i_{2}} \cdots a_{i_{n}}\right) \cdot \overline{\gamma_{n}}$, where $\overline{\gamma_{n}}:=\left(1-\widetilde{t}_{1,2}\right)\left(1-\widetilde{t}_{1,3}\right) \cdots\left(1-\widetilde{t}_{1, n}\right) \in \mathcal{K}_{n}$.

ii) $a_{i_{1}} a_{i_{2}} \cdots a_{i_{n}}=Y_{i_{1} \cdots i_{n}} \cdot{\overline{\gamma_{n}}}^{-1}$, with

$$
{\overline{\gamma_{n}}}^{-1}=\sum_{\pi \in S_{n}} \tilde{\pi} \cdot \prod_{\pi(i)>\pi(i+1)} Q_{\{1, \ldots, i\}} /\left(1-Q_{\{1,2\}}\right) \cdots\left(1-Q_{\{1, \ldots, n\}}\right)
$$

iii) The set $\left\{Y_{\mathbf{i} \cdot \tilde{\pi}} \mid \tilde{\pi} \in H \backslash S_{n}\right\},\left(H=S t_{\mathbf{i}}\right)$ is a linearly independent set if $\left|q_{i_{r} i_{s}}\right|<1$, $1 \leq r \neq s \leq n$.

Proof. i) The formula (22) can be rewritten as

$$
Y_{i_{1} i_{2} \cdots i_{n}}=a_{i_{1}} a_{i_{2}} \cdots a_{i_{n}}\left(1-q_{i_{2} i_{1}} t_{1,2}\right)\left(1-q_{i_{3} i_{1}} q_{i_{3} i_{2}} t_{1,3}\right) \cdots\left(1-q_{i_{n} i_{1}} q_{i_{n} i_{2}} \cdots q_{i_{n} i_{n-1}} t_{1, n}\right)
$$

By using $\widetilde{t}_{1, l}=Q_{l, 1} \cdots Q_{l, l-1} t_{1, l}=t_{1, l} Q_{1,2} Q_{1,3} \cdots Q_{1, l}$ the claim i) follows.

ii) The proof of ii) is similar to that of Proposition.2.1.1 in [10].

iii) Follows from ii). 
PROPOSITION 2. The $Y_{\mathbf{i}}$ 's satisfy the following (twisted) differential equations:

$$
\begin{aligned}
\text { i) }{ }_{l} \partial\left(Y_{i_{1} \cdots i_{n}}\right)^{\dagger} & =\sum_{\left(j \geq 2: i_{j}=l\right)} d_{i_{1} \cdots i_{n}}^{(j)}\left(Y_{i_{1} \cdots i_{j} \cdots i_{n}}\right)^{\dagger}, \quad(n \geq 2) \\
\text { ii) } \quad{ }_{l} \partial Y_{i_{1}}^{\dagger} & =\delta_{i_{1} l}, \quad(n=1)
\end{aligned}
$$

where ${ }_{l} \partial$ is defined in (21), and where

$$
d_{i_{1} \cdots i_{n}}^{(j)}:=q_{i_{j} i_{j+1}} \cdots q_{i_{j} i_{n}}\left(1-\left|q_{i_{j} i_{1}} \cdots q_{i_{j} i_{j-1}}\right|^{2}\right)
$$

Proof. By induction. For $n=2$ we have $Y_{i_{1} i_{2}}=\left[a_{i_{1}}, a_{i_{2}}\right]_{a_{i_{2} i_{1}}}=a_{i_{1}} a_{i_{2}}-q_{i_{2} i_{1}} a_{i_{2}} a_{i_{1}}$ what implies $\left(Y_{i_{1} i_{2}}\right)^{\dagger}=a_{i_{2}}^{\dagger} a_{i_{1}}^{\dagger}-q_{i_{1} i_{2}} a_{i_{1}}^{\dagger} a_{i_{2}}^{\dagger}$ (here we use $\left.\left(q_{i j}\right)^{*}=q_{j i}\right)$. Hence

$$
\begin{aligned}
{ }_{l} \partial\left(Y_{i_{1} i_{2}}\right)^{\dagger} & =\delta_{l i_{2}} a_{i_{1}}^{\dagger}+\delta_{l i_{1}} q_{l i_{2}} a_{i_{2}}^{\dagger}-q_{i_{1} i_{2}}\left(\delta_{l i_{1}} a_{i_{2}}^{\dagger}+\delta_{l i_{2}} q_{l i_{1}} a_{i_{1}}^{\dagger}\right) \\
& =\left(1-q_{i_{1} i_{2}} q_{i_{2} i_{1}}\right) a_{i_{1}}^{\dagger} \delta_{l i_{2}}=d_{i_{1} i_{2}}^{(2)} Y_{i_{1}}^{\dagger} \delta_{l i_{2}}
\end{aligned}
$$

Now we suppose that (25) holds true for $n-1$. Then, from (19) it follows that

$$
\begin{gathered}
{ }_{l} \partial\left(Y_{i_{1} \cdots i_{n}}\right)^{\dagger}={ }_{l} \partial\left[a_{i_{n}}^{\dagger}\left(Y_{i_{1} \cdots i_{n-1}}\right)^{\dagger}-q_{i_{1} i_{n}} \cdots q_{i_{n-1} i_{n}}\left(Y_{i_{1} \cdots i_{n-1}}\right)^{\dagger} a_{i_{n}}^{\dagger}\right] \\
=\delta_{l i_{n}}\left(Y_{i_{1} \cdots i_{n-1}}\right)^{\dagger}+q_{l i_{n}} a_{i_{n} l}^{\dagger} \partial\left(Y_{i_{1} \cdots i_{n-1}}\right)^{\dagger} \\
-q_{i_{1} i_{n}} \cdots q_{i_{n-1} i_{n}}\left[l \partial\left(Y_{i_{1} \cdots i_{n-1}}\right)^{\dagger} a_{i_{n}}^{\dagger}+q_{i_{n} i_{1}} \cdots q_{i_{n} i_{n-1}-1} \delta_{l i_{n}}\left(Y_{i_{1} \cdots i_{n-1}}\right)^{\dagger}\right] \\
=\delta_{l i_{n}}\left(1-\left|q_{i_{1} i_{2}} \cdots q_{i_{n-1} i_{n}}\right|^{2}\right)\left(Y_{i_{1} \cdots i_{n-1}}\right)^{\dagger}+ \\
\sum_{j=2 ; i_{j}=l}^{n-1} q_{l i_{n}} d_{i_{1} \cdots i_{n-1}}^{(j)}\left[a_{i_{n}}^{\dagger}\left(Y_{i_{1} \cdots \hat{i}_{j} \cdots i_{n-1}}\right)^{\dagger}-q_{i_{1} i_{n}} \cdots \widehat{q_{l i_{n}}} \cdots q_{i_{n-1} i_{n}}\left(Y_{i_{1} \cdots \hat{i}_{j} \cdots i_{n-1}}\right)^{\dagger} a_{i_{n}}^{\dagger}\right] \\
=\delta_{l i_{n}} d_{i_{1} \cdots i_{n}}^{(n)}\left(Y_{i_{1} \cdots i_{n-1}}\right)^{\dagger}+\sum_{j=2 ; i_{j}=l}^{n-1} d_{i_{1} \cdots i_{n}}^{(j)}\left(Y_{i_{1} \cdots \hat{i}_{j} \cdots i_{n}}\right)^{\dagger} \\
=\sum_{n \geq j \geq 2 ; i_{j}=l} d_{i_{1} \cdots i_{n}}^{(j)}\left(Y_{i_{1} \cdots i_{j} \cdots i_{n}}\right)^{\dagger} .
\end{gathered}
$$


This completes the proof of Proposition 2.

Now we proceed with solving (20) to get $X_{\mathbf{i}^{-}}$-components of our number operator $N_{k}$. There are two approaches:

The first approach, developed in [8], is based on an observation that in (37) the index $i_{1}$ survives in all terms of the r.h.s. So, we could look for $X_{\mathbf{i}}$ 's in the form of a linear combination of such $Y_{\mathbf{i}}$ 's with the first index fixed $\left(=k\right.$ for $\left.N_{k}\right)$.

$$
\left(X_{\mathbf{i}}\right)^{\dagger}=\sum_{\mathbf{j}=\mathbf{i} . \pi, \pi \in S_{1} \times S_{n-1}}\left(Y_{\mathbf{j}}\right)^{\dagger} c_{\mathbf{j}, \mathbf{i}}
$$

By applying the twisted derivative ${ }_{l} \partial$ to (39), the left hand side gives

$$
\begin{gathered}
{ }_{l} \partial\left(X_{\mathbf{i}}\right)^{\dagger}=\left(X_{i_{1} \cdots i_{n-1}}\right)^{\dagger} \delta_{l i_{n}}(b y(20)) \\
=\sum_{\sigma \in S_{1} \times S_{n-2}}\left(Y_{\left.i_{\sigma(1)} \cdots i_{\sigma(n-1)}\right)}\right)^{\dagger} c_{i_{\sigma(1)} \cdots i_{\sigma(n-1)}, i_{1} \cdots i_{n-1}} \delta_{l i_{n}} \quad(b y(39)) .
\end{gathered}
$$

The ${ }_{l} \partial$ applied to the right hand side of (39) gives:

$$
\begin{gathered}
\sum_{\pi \in S_{1} \times S_{n-1}} l \partial\left(Y_{\mathbf{i} \cdot \pi}\right)^{\dagger} c_{\mathbf{i} \cdot \pi, \mathbf{i}} \\
=\sum_{\pi \in S_{1} \times S_{n-1}} \sum_{(n \geq j \geq 2 ; l=\pi(j))} d_{\mathbf{i} . \pi}^{(j)}\left(Y_{i_{\pi(1)} \cdots i_{\pi(j)} \cdots i_{\pi(n)}}\right)^{\dagger} c_{\mathbf{i} \cdot \pi, \mathbf{i}}(b y(37)) .
\end{gathered}
$$

By linear independence of $Y_{\mathbf{i}}$ 's (cf. Corollary 1) we obtain the following system of $(n-1)$ ! equations (in the generic case) for $(n-1)$ ! unknown coefficients $c_{\mathbf{i} . \pi, \mathbf{i}}$, $\left(i_{1}=k, \pi \in S_{1} \times S_{n-1}\right)$ :

EQUATIONS FOR $c_{\mathbf{j}, \mathbf{i}}$ 's:

$$
\sum_{n \geq j \geq 2} d_{\mathbf{i} . \pi t_{j, n}}^{(j)} c_{\mathbf{i} . \pi t_{j, n}, \mathbf{i}}=\delta_{\pi(n), n} c_{(\mathbf{i} . \pi)^{\prime}, \mathbf{i}^{\prime}}
$$

where $\pi \in S_{1} \times S_{n-1}, t_{j, n}$ denotes the cyclic permutation which sends $1,2, \ldots, j, j+$ $1, \ldots, n$ to $1,2, \ldots, n, j, \ldots, n-1$ and $\mathbf{i}^{\prime}=i_{1} \ldots i_{n-1}$. 
Note that our derivation of the equations (40) (generic case) will yield (by summation) the equations for the nongeneric case (i.e. when there are repetitions among $\left.i_{1}, \cdots, i_{n}{ }^{\prime} s\right)$. This justifies the form of our expression (15) for the number operators $N_{k}{ }^{\prime} s$.

The second approach to solving the recursive system (20) for $X_{\mathbf{i}}$ 's is to write $Y_{\mathbf{i}}$ 's in terms of $X_{\mathbf{i}}$ 's, again with the first index fixed $\left(=k\right.$ for $\left.N_{k}\right)$.

$$
\left(Y_{\mathbf{i}}\right)^{\dagger}=\sum_{\mathbf{j}=\mathbf{i} . \pi, \pi \in S_{1} \times S_{n-1}}\left(X_{\mathbf{j}}\right)^{\dagger} e_{\mathbf{j}, \mathbf{i}}
$$

PROPOSITION 3. The coefficients $e_{\mathbf{j}, \mathbf{i}}$ satisfy the following recursions:

$$
e_{\mathbf{i} . \pi, \mathbf{i}}=d_{\mathbf{i}}^{(r)} e_{\mathbf{i}^{\prime}, \pi^{\prime}, \mathbf{i}^{\prime}}
$$

where

$r=\pi(n), \mathbf{i}^{\prime}=i_{1} \ldots i_{n-1}, \pi^{\prime}=t_{r, n} \pi\left(\Rightarrow \pi=t_{r, n}^{-1} \pi^{\prime}, \pi^{\prime} \in S_{n-1}\right)$, and $d_{\mathbf{i}}^{(r)}=d_{i_{1} \cdots i_{n}}^{(r)}$ is defined in (38).

Proof. By applying ${ }_{l} \partial$ to both sides of (41), and using (37), we obtain

$$
\begin{array}{r}
\sum_{\mathbf{j}=\mathbf{i} . \pi, \pi \in S_{1} \times S_{n-1}}\left(X_{j_{1} \ldots j_{n-1}}\right)^{\dagger} e_{\mathbf{j}, \mathbf{i}} \delta_{l, j_{n}}=\sum_{r \geq 2, i_{r}=l} d_{\mathbf{i}}^{(r)}\left(Y_{i_{1} \ldots \hat{i}_{r} \ldots i_{n}}\right)^{\dagger} \\
=\sum_{r \geq 2, i_{r}=l} d_{\mathbf{i}}^{(r)} \sum_{\sigma \in S_{1} \times S_{n-2}}\left(X_{\mathbf{i}_{\hat{r}} . \sigma}\right)^{\dagger} e_{\mathbf{i}_{\hat{r}}, \sigma, \mathbf{i}_{\hat{r}}}
\end{array}
$$

where $\mathbf{i}_{\hat{r}}:=i_{1} \ldots i_{r-1} i_{r+1} \ldots i_{n}$. Observe that $i_{\pi(1)} \ldots i_{\pi(n-1)}=\mathbf{i}_{\hat{r}} . \sigma$ iff $r=\pi(n)$ and $\sigma=t_{r, n} \pi$

By equating the coefficients in (43) and (44) the proof of Proposition 3. follows. 
Note that the recursion (42) corresponds to the multiplication by the following element (of the twisted group algebra):

$$
\eta_{n}:=\sum_{k=2}^{n} Q_{\{k, k+1\}} \cdots Q_{\{k, n\}}\left(1-Q_{\{k, 1\}} \cdots Q_{\{k, k-1\}}\right) \tilde{t}_{k, n}^{-1} .
$$

Let $E=\left(e_{\mathbf{i}, \mathbf{j}}\right)$, with $i_{1}=j_{1}(=k)$ fixed,be the $(n-1) ! \times(n-1) !$ transition matrix (in the generic case), with entries $e_{\mathbf{i}, \mathbf{j}}$ from (41). In [8] the linear equations for the entries of $E^{-1}$ are constructed for general $n$ and solved in special cases for $n=1,2,3$. From these computations it was conjectured (in [8]) that $E^{-1}$ is related to the inverse of the Gram matrix $A$, see eq. (3); here we prove this conjecture.

By comparing $\xi_{n}$ from (36) with $\eta_{n}$ from (42a) we get

$$
w_{n} \eta_{n} w_{n}=\xi_{n}
$$

and we deduce the following:

LEMMA 2. The matrix $E$ is the matrix of the right multiplication by the following element of our twisted group algebra $K_{n}{ }^{\sim}\left[S_{n}\right]$ :

$$
w_{n} \widetilde{\alpha}_{n-1,1} \widetilde{\delta}_{n} w_{n}
$$

Here $w_{n}=n \ldots 21$ denotes the longest element in $S_{n}$.

Proof. Follows by iteratively applying the result of Lemma 1, using the definition (32) of $\tilde{\delta}_{n}$ together with the recursions obtained in the Proposition 3.

5. The main results. Now we prove the following theorem:

THEOREM 1. The number operators in the multiparameter quon algebra $\mathcal{A}^{(\mathbf{q})}$ eq. (1) are given, in the expanded form, by: 


$$
N_{k}=a_{k}^{\dagger} a_{k}+\sum_{n=1}^{\infty} \sum_{\mathbf{i}, i_{1}=k} \sum_{\pi \in S_{1} \times S_{n-1}} \hat{A}_{\mathbf{i}, \mathbf{i} . \pi}^{-1}\left(Y_{\mathbf{i} . \pi}\right)^{\dagger} Y_{\mathbf{i}}
$$

where the matrix $\hat{A}$ denotes the matrix obtained from the Gram matrix $A=$ $\oplus_{n \geq 0} \oplus_{k_{1} \leq \cdots \leq k_{n}} A^{k_{1} \ldots k_{n}}$ (described in (4)) by replacing each block $A^{k_{1} \ldots k_{n}}\left(k_{1} \leq \cdots \leq\right.$ $\left.k_{n}\right)$ with a specialized $n ! \times n$ ! block $\left.A^{12 \cdots n}\right|_{1 \mapsto k_{1}, 2 \mapsto k_{2} \cdots n \mapsto k_{n}}$ and $Y_{\mathbf{i}}$ are given by (22).

Or, in the reduced form, by:

$$
N_{k}=a_{k}^{\dagger} a_{k}+\sum_{n=1}^{\infty} \sum_{\mathbf{i}, i_{1}=k} \sum_{\tilde{\pi} \in S t a b_{\mathbf{i}} \backslash S_{1} \times S_{n-1}} \tilde{A}_{\mathbf{i}, \mathbf{i} . \tilde{\pi}}^{-1}\left(Y_{\mathbf{i} . \tilde{\pi}}\right)^{\dagger} Y_{\mathbf{i}}
$$

where the reduction procedure is given with respect to the groups $S_{1} \times S_{n-1}$ (instead of $S_{n}$ ) analogously to the reduction procedure described in the text preceding (6).

The proof of this theorem relies on one more lemma.

LEMMA 3. We have

The $S_{1, n-2,1}$-component of $\tilde{\alpha}_{n}^{-1}$

$=$ The $S_{1, n-2,1}$-component of $\widetilde{\delta}_{n}^{-1} \times \widetilde{\alpha}_{n-1,1}^{-1}$

Proof of Lemma 3.: This is a generalization of a Zagier's result [15]. Here we sketch the proof. By observing that $\widetilde{\alpha}_{n-1}=\widetilde{\alpha}_{n-1,1}$ we can write (c.f.(33))

$$
\begin{gathered}
\widetilde{\alpha}_{n}=\widetilde{\alpha}_{n-1,1} \widetilde{\delta}_{n} \widetilde{\gamma}_{n}^{-1} \\
\widetilde{\alpha}_{n}^{-1}=\widetilde{\gamma}_{n} \widetilde{\delta}_{n}^{-1} \widetilde{\alpha}_{n-1,1}^{-1}
\end{gathered}
$$

where, according to (31),

$$
\tilde{\gamma}_{n}=\left(1-\tilde{t}_{1, n}\right)\left(1-\widetilde{t}_{2, n}\right) \cdots\left(1-\tilde{t}_{n-1, n}\right)=\sum_{k=1}^{n}(-1)^{n-k} \sum_{\pi \in S_{n, k}} \tilde{\pi}^{-1}
$$


with $S_{n, k} \subset S_{n}$ denoting the set of all permutations such that $\pi(1)<\cdots<\pi(k)=$ $n>\cdots>\pi(n)$. Note that $\widetilde{\delta}_{n}$ involves only permutations belonging to $S_{n-1} \times S_{1}$ (c.f. (32); for an explicit formula for the inverse of $\widetilde{\delta}_{n}$ see Proposition 2.1.1. in [10]). Now it is clear that only the trivial term in $\tilde{\gamma}_{n}$ can contribute to the $S_{1, n-2,1}$-component of $\tilde{\alpha}_{n}^{-1}$. The Lemma 3 is proved. This establishes the connection between $E^{-1}$ and the inverse $A^{-1}$ of the Gram matrices.

Proof of Theorem 1.By using Lemmas 2 and 3, together with the symmetry property (9) and hermiticity (8) of the multiparameter Zagier matrices, we obtain

$$
X_{\mathbf{i}}^{\dagger}=\sum_{\pi \in S_{1} \times S_{n-1}} Y_{\mathbf{i} . \pi}^{\dagger} A_{\mathbf{i}, \mathbf{i} . \pi}^{-1}
$$

in expanded form, and similarly

$$
X_{\mathbf{i}}^{\dagger}=\sum_{\tilde{\pi} \in H \backslash S_{1} \times S_{n-1}} Y_{\mathbf{i} . \tilde{\pi}}^{\dagger} A_{\mathbf{i}, \mathbf{i} . \tilde{\pi}}^{-1}
$$

in reduced form. This completes the proof of Theorem 1. The method for calculating the inverse of the matrix $A$ is explained in [9, Theorem 2.2.17].

COROLLARY 2. Let us assume infinite set I of indices, then the number operator $N_{k}$ restricted to the finite subset $I_{f} \subseteq I$ is obtained from eq. (46), eq. (47) by projecting out all words with letters from the subset $I_{f}$. Specially if $I_{f}=\{k\}$ we recover the simple formula for $N_{k}$ for a single oscillator obtained by Greenberg ([2], [3]).

Also, if we plug into (46) and (47) the formulas (22) expressing $Y_{\mathbf{i}}^{\prime} \mathbf{s}$ in terms of monomials we obtain Zagier or Stanciu type formulas for number operator.

The transition operators will be considered in the near future. 


\section{Acknowledgments.}

This work was supported by the Ministry of Science and Technology of the Republic of Croatia under contract No. 0098003 and 037009. 


\section{REFERENCES}

[1] Bardek V.,Meljanac S.,Perica A.: Generalized statistics and dynamics in curved spacetime.Phys.Lett.B338, 20-22 (1994);

[2] Greenberg O.W.: Example of infinite statistics. Phys.Rev.Lett.64, 705-708 (1990);

[3] Greenberg O.W.: Particles with small violations of Fermi or Bose statistics. Phys.Rev.D43, 4111-4120 (1991);

[4] Greenberg O.W.: Interactions of particles having small violations of statistics. Physica A180, 419-427 (1992);

[5] Mohapatra R.N.: Infinite statistics and a possible small violation of the Pauli principle. Phys.Lett.242B, 407-410 (1990);

[6] Møller J.S.: Second quantization in a quon algebra. J.Phys.A:Math.Gen. 26, 4643-4562 (1993);

[7] Meljanac S.,Perica A.: Generalized quon statistics. Mod.Phys.Lett.A9, 3293-3299 (1994);

[8] Meljanac S.,Perica A.: Number operators in a general quon algebra. J.Phys.A:Math.Gen.27, 4737-4744 (1994);

[9] Meljanac S.,Svrtan D.: Study of Gram matrices in Fock representation of multiparametric canonical commutation relations, extended Zagier's conjecture, hyperplane arrangements and quantum groups. Math.Commun.1, 1-24 (1996); [10] Meljanac S.,Svrtan D. Determinants and inversion of Gram matrices in Fock representation of $\left\{q_{k l}\right\}$-canonical commutation relations and applications to hyperplane arrangements and quantum groups. Proof of an extension of Zagier's 
conjecture. Preprint RBI-TH-5/Nov.1995;

[11] Stanciu S.: The Energy Operator for Infinite Statistics. Commun.Math,Phys. 147, 211-216 (1992);

[12] Varchenko A.: Bilinear Form of Real Configuration of Hyperplanes. Advances in Mathematics 97, 110-144 (1993);

[13] Werner R.F.: The free quon gas suffer Gibbs' paradox. Phys.Rev.D48, 2929-2934 (1993);

[14] Wu Z., Yu T.: Construction of Bose and Fermi operators in terms of $q=0$ quon operators. Phys.Lett.A179, 266-270 (1993);

[15] Zagier D.: Realizability of a model in infinite statistics, Commun.Math.Ph ys.147, 199-210 (1992);

[16] Božejko M. and Speicher R.: Completely positive maps on Coxeter groups, deformed commutation relations, and operator spaces. Math.Ann. 300, 97-120 (1994);

[17] Jørgensen P.E.T., Schmitt L.M., Werner R.F.: Positive representations of general commutation relations allowing Wick ordering. J.-Funct.-Anal. 134, 33-99 (1995); 